\title{
20p13 microdeletion syndrome
}

INSERM

\section{Source}

INSERM. (1999). Orphanet: an online rare disease and orphan drug data base. 20p13 microdeletion syndrome. ORPHA:313781

20 13 microdeletion syndrome is a rare chromosomal anomaly characterized by developmental delay, mild to moderate intellectual disability, epilepsy, and unspecific dysmorphic signs. High palate, delayed permanent tooth eruption, hypoplastic fingernails, clinodactyly and short fingers have also been reported. 